\title{
Severe Measles Encephalitis and Acute Disseminated Encephalomyelitis in an Immunocompetent Japanese Young Male
}

\author{
Yoshikazu Mutoh ${ }^{1}$, Yuya Kano른 Takuya Oguri ${ }^{2}$, Hideki Kato², Takumi Umemura ${ }^{2}$, \\ Chihiro Norizuki ${ }^{3}$, Toshihiko Ichihara ${ }^{3}$ and Hiroyuki Yuasa ${ }^{2}$
}

\begin{abstract}
:
Measles encephalitis rarely affects young adults and has no established treatment strategy. This brief report described the rare case of an immunocompetent 30-year-old man with severe measles pneumonia and encephalitis, following the autoimmune disease acute disseminated encephalomyelitis, during a large measles outbreak in 2018 in Japan. With multidisciplinary treatments, including corticosteroids, intravenous immunoglobulins, vitamin A, and therapeutic plasma exchange, the patient was successfully treated. This case provides a new strategy for treating measles encephalitis and its complications during measles outbreak.
\end{abstract}

Key words: measles, outbreak, therapeutic plasma exchange, encephalitis, ADEM

(Intern Med 60: 1971-1976, 2021)

(DOI: 10.2169/internalmedicine.5362-20)

\section{Introduction}

In 2015, the World Health Organization (WHO) listed Japan as one of the countries that had successfully eliminated measles, a feat accomplished via implementing a national program that stopped viral transmission (1). Globally, owing to the achievement of measles vaccine coverage of $>95 \%$, the herd immunity was boosted. The annual global incidence of measles and related deaths had decreased by $87 \%$ and 84\%, respectively, between 2000 and 2016 (2). However, in 2018, large measles outbreaks occurred worldwide, mainly involving unvaccinated or partially vaccinated children. There has been a recent rise in the international incidence of measles cases, with $173,000,320,000$, and $>440,000$ cases reported in 2017, 2018, and 2019, respectively (3). The last worldwide outbreak is still ongoing.

Currently, most of the patients recover without any treatment; on the other hand, around one-fifth need hospitalization, and approximately $30 \%$ experience complications. Moreover, an estimated 1-3/1,000 can develop measles encephalitis, affecting adults more commonly than it does chil- dren (4).

In 2018, starting with the index case of an Asian traveler, a large outbreak of measles occurred, mainly among young adults in Japan. During this outbreak, $>170$ individuals were reportedly infected, especially in Okinawa. This report describes our successful management of a patient with severe measles encephalitis, which was treated using corticosteroids, intravenous immunoglobulins, vitamin A, and therapeutic plasma exchange (TPE).

\section{Case Report}

A 30-year-old Japanese presented to a hospital in May 2018 with nasal discharge, cough, diarrhea, joint pain, fever, conjunctivitis, and a generalized maculopapular rash 5 days earlier. He had no significant past medical history and had reportedly been vaccinated against measles only once in his childhood. A serum measles polymerase chain reaction (PCR) test was performed considering the ongoing measles outbreak at the time. The result was positive, and acetaminophen was prescribed.

He visited our emergency room the next day owing to

${ }^{1}$ Department of Infectious Diseases, Tosei General Hospital, Japan, ${ }^{2}$ Department of Neurology, Tosei General Hospital, Japan and ${ }^{3}$ Department of Infection Control Team, Tosei General Hospital, Japan

Received: May 16, 2020; Accepted: November 23, 2020; Advance Publication by J-STAGE: January 15, 2021

Correspondence to Dr. Yoshikazu Mutoh, infection-travel@tosei.or.jp 


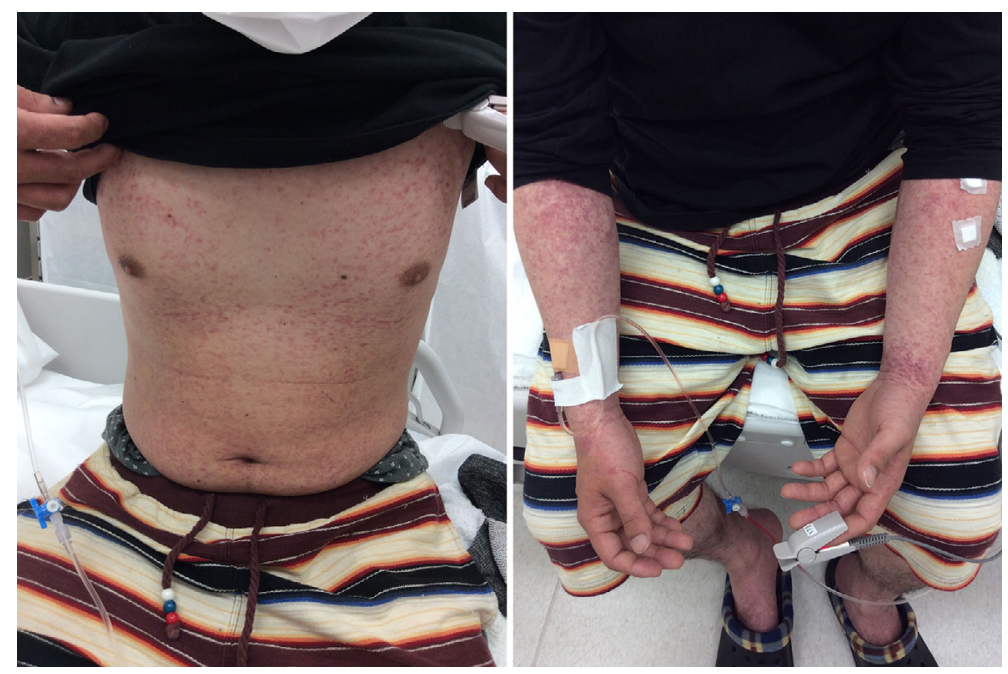

Figure 1. Rash in the trunk and extremities on the first day of admission.

symptomatic worsening and development of a high-grade fever. On initial examination, he was alert. His vital signs were as follows: temperature, $39.5^{\circ} \mathrm{C}$; heart rate, 93 beats/ min; blood pressure, $117 / 55 \mathrm{mmHg}$; respiratory rate, 24 breaths/min; and oxygen saturation, 97\% (ambient). Physical examination revealed conjunctivitis and a characteristic, diffuse, maculopapular rash involving the face, extremities, and trunk (Fig. 1). Blood tests showed a white blood cell count of $10,200 / \mathrm{mm}^{3}$ (66\% neutrophils, $21 \%$ lymphocytes, and $7 \%$ monocytes) and a platelet count of $141,000 / \mathrm{mm}^{3}$. Lactate dehydrogenase and C-reactive protein levels were 1,038 IU/L and $6.42 \mathrm{mg} / \mathrm{dL}$, respectively. Chest X-ray revealed consolidation in the left upper lung. In addition, his HIV, HCV, and HBV status was negative. The patient was diagnosed with measles pneumonia and admitted to our intensive care unit; because of the possibility of secondary bacterial infection, ceftriaxone ( $2 \mathrm{~g} /$ day) was initiated.

The patient's level of consciousness suddenly deteriorated on the second day of admission. His Glasgow Coma Scale (GCS) score was 7, and numbness of the legs and loss of the pupillary light reflex was noted. A lumbar puncture was performed, with the following results: cerebrospinal fluid (CSF) pressure $>270 \mathrm{mmH}_{2} \mathrm{O}$; total cell count, $1,858 / \mu \mathrm{L}$ (75\% lymphocytes), and glucose level, $35 \mathrm{mg} / \mathrm{dL}$ (blood glucose, $149 \mathrm{mg} / \mathrm{dL}$ ). The measles specific $\operatorname{IgM}$ antibody was detected in his CSF, and 3 weeks later, it also appeared in his blood through laboratory analysis using enzymelinked immuno-sorbent assay (ELISA). However, measlesspecific IgG antibody was not detected in both tests.

Brain magnetic resonance imaging (MRI) revealed a remarkably high-intensity splenial lesion in the corpus callosum on T2-weighted fluid-attenuated inversion recovery images and diffusion-weighted images (Fig. 2). Therefore, we administered intravenous acyclovir (1,500 mg/day), methylprednisolone (mPSL) pulse therapy $(1,000 \mathrm{mg} / \mathrm{day})$, and intravenous immunoglobulin G (IVIG) (5,000 mg/day), as well as an intravenous glycerol solution to suppress cerebral edema. However, the patient's consciousness level worsened on the third day of admission (GCS score, 3), and he had to be intubated.

After intubation and owing to extremely low levels of serum vitamin A (102 $\mu \mathrm{g} / \mathrm{dL}$; range, 431-1,042 U), we administered intravenous vitamin A (200,000 U/day) for two days, followed by switching to another antibiotic (tazobactam/ piperacillin, $18 \mathrm{~g}$ /day). Although the rash gradually resolved (Fig. 3), his level of consciousness did not improve, even after mPSL pulse therapy and IVIG therapies. On day 8 , his limbs were completely paralyzed, and deep tendon reflexes of extremities were disappeared. We performed nerve conduction study and it was revealed that the distal latency was prolonged and compound muscle action potential amplitude was decreased of the left median nerve. The frequency of Fwaves in the left tibial nerve was prolonged and the frequency decreased. On day 12, a tracheostomy was performed, and the patient was transferred to the neurology ward. Two additional consecutive mPSL pulse and IVIG therapies were administered; however, his temperature was still elevated, and diffusion-weighted MRI of the brain revealed new high-density areas in the caudate and thalamus on day 30 (Fig. 2). Additionally, the patient was positive for serum antiganglioside antibodies (IgM GM1 antibody and GalNac-GD1a antibody), and the CSF myelin basic protein level was $19,400 \mathrm{pg} / \mathrm{mL}$.

Considering these findings and the patient's persistent unconsciousness, we suspected secondary Guillain-Barré syndrome or acute disseminated encephalomyelitis (ADEM). Therefore, on day 39, TPE was begun. Seven consecutive TPE procedures (2/week) were performed, with 40 units of plasma volume exchanged per procedure.

After the first TPE session, the patient's motor functions gradually recovered, and his liver enzyme levels and temperature decreased. Brain MRI performed on day 51 revealed disappearance of abnormal high intensity signals on DWI, and the patient gradually regained the ability to move spontaneously. Measles virus PCR tests of blood, urine, and throat swab specimens were performed monthly; they be- 

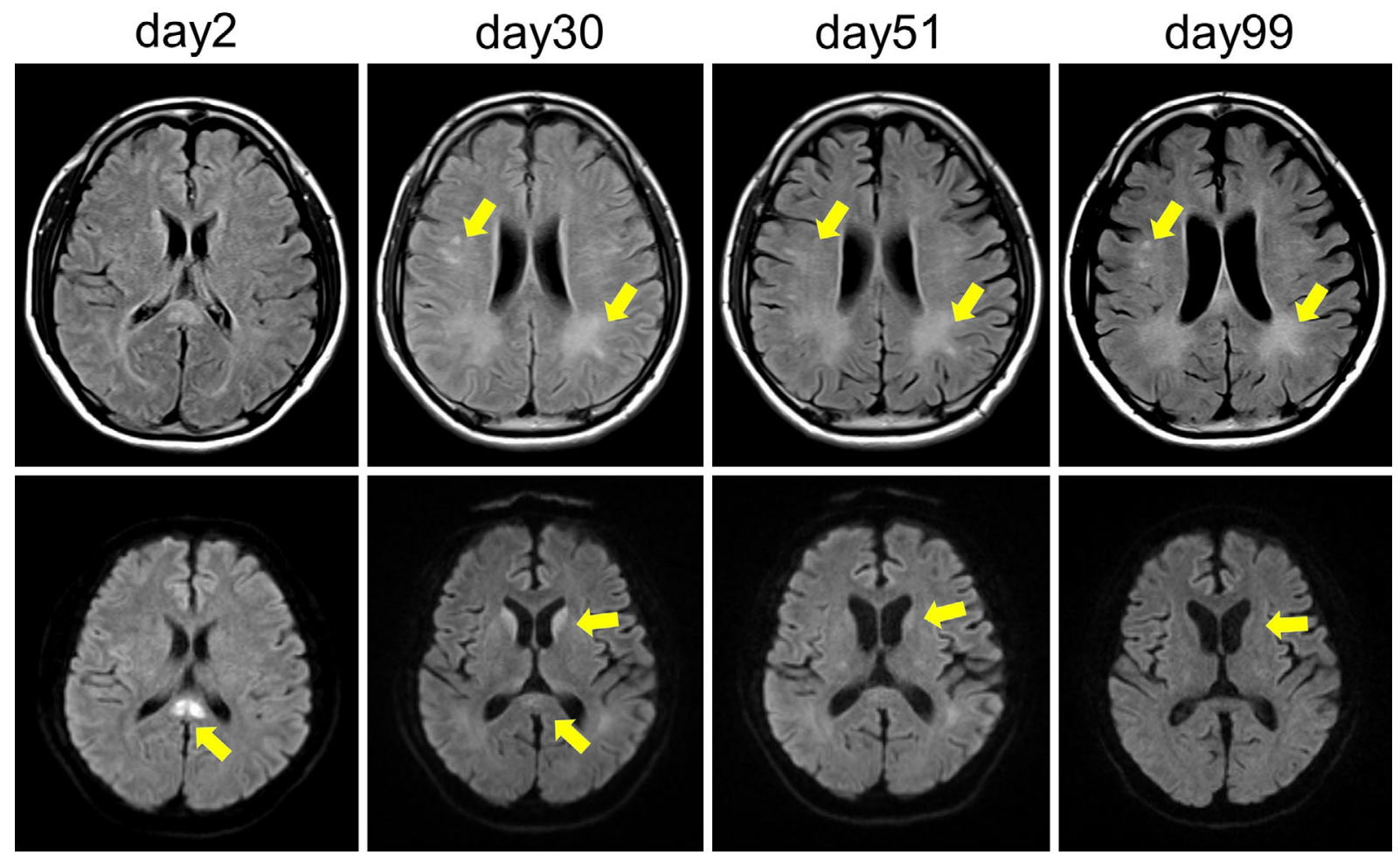

Figure 2. MRI findings during the clinical course. Upper row: T2-weighted fluid-attenuated inversion recovery images depicted scattered high signal intensity areas in the white matter (arrows). Lower row: Diffusion-weighted images display high-intensity areas in the caudate putamen, thalamus, and splenium of the corpus callosum (arrows).
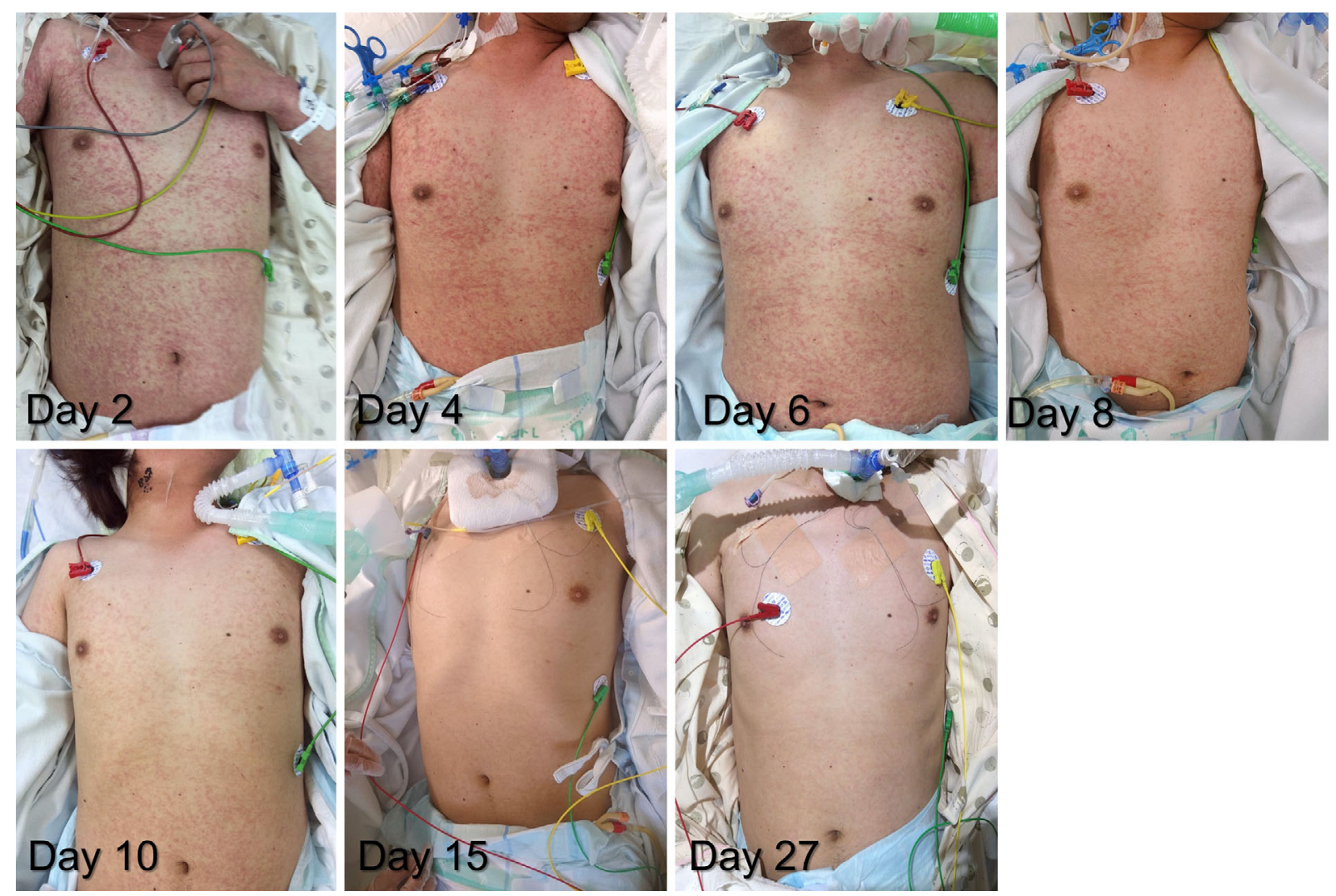

Figure 3. Clinical improvement of rash in the trunk. 


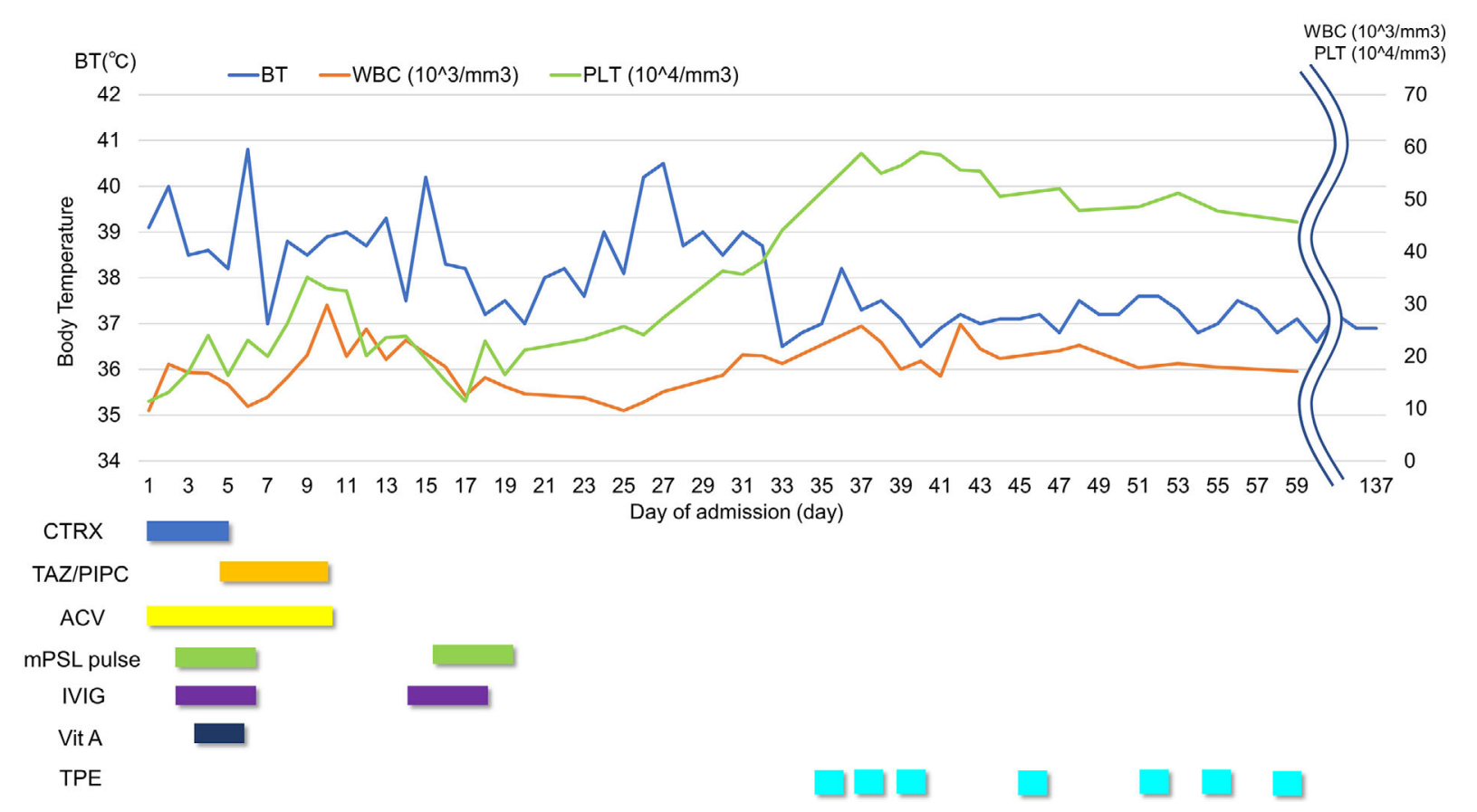

Figure 4. The Clinical course of the patient during the first 60 days (Total duration of hospitalization was 137 days).

came negative on day 88 , after which patient isolation (to prevent airborne transmission) was discontinued. Finally, on day 137 , he fully regained consciousness and was able to walk and eat independently; at this point, he was transferred to a long-term care facility (Fig. 4). The causative genotype of the measles virus was identified as type D8, which was the same as that of the pathogen that had triggered the ongoing 2018 outbreak in Okinawa.

\section{Discussion}

Herein, we described a patient with measles encephalitis saved by our multidisciplinary management, which occurred during an outbreak of 2018 in Japan (primarily January to April). The index case of the 2018 measles outbreak in Japan was inferred to be a traveler from Taiwan, who was infected with measles in Thailand. He visited Okinawa, the southernmost prefecture in Japan, while he was sick with fever and rash. Consequently, $>100$ individuals in Okinawa were infected, $80 \%$ of whom were $<40$-years-old (5). In the early part of April, the first patient with measles in Aichi was confirmed to have traveled to Okinawa during the outbreak. Our patient, who lives in Aichi and has never been to Okinawa, became symptomatic on the middle of May and was diagnosed five days later. Interestingly, he had no known contact with any other measles patients. Nevertheless, his measles viral strain was identified as type D8, which was the same as that of the causative pathogen of the outbreak in Japan. He had received only one dose of measles vaccine in his childhood. The administration of 2 doses of measles vaccine has been part of Japan's national immunization program since 1990. Currently, the occurrence of measles in Japan decreased by $>90 \%$. However, compared with a 2-dose measles vaccination rate of $>80 \%$ among children, less than $20 \%$ of the adult population has been similarly inoculated (6). This disparity might contribute to the development of measles outbreaks. Therefore, implementing effective and widespread vaccination programs is urgently required to eliminate measles.

Our patient developed pneumonia and neurological symptoms 8 days after onset, and measles RNA was isolated from his CSF. Although it is quite rare, we considered the possibility that both the measles subclinical central nerve involvement and pneumonia had started concurrently with the early phase of measles infection. Measles complications are more severe in adults, particularly in malnourished and immunocompromised individuals (7). However, our patient had no significant relevant medical history.

In general, there are 4 categories of measles-associated neurological complications depending on their presentation: primary measles encephalitis, acute post-measles encephalitis, measles inclusion-body encephalitis, and subacute sclerosing panencephalitis (8). Our patient was thought to have primary measles encephalitis or acute post-measles encephalitis based on his clinical course. Both complications affect 1-3/1,000 patients with measles, have high mortality rates, and cause neurological symptoms during the early stages of infection owing to the invasion of the central nervous system by measles virus.

Our patient also had ADEM, as determined via MRI. ADEM is an abnormal immune reaction with autoimmune demyelination of the central nervous system due to febrile viral infections or vaccinations (9). Its diagnosis is based on the clinical history and neurological evaluation, including 
neuroimaging. The occurrence of ADEM among measles patients is rare $(1 / 1,000$ cases) but can develop within days to weeks of the initial infection (10) and is often fatal. In our knowledge, our patient was the only case of measles encephalitis with ADEM during the 2018 outbreak in Japan.

The etiology of measles-associated ADEM remains unclear. Rafat et al reported four patients with measles encephalitis, including three young patients without any underlying comorbid condition, explained as demyelination mediated by a hyperactive autoimmune process, resembling ADEM (11). This destruction is believed to be demonstrated by lymphoproliferative response and against myelin basic protein in the CSF (12). Our patient also demonstrated lymphocytic pleocytosis and myelin basic protein in CSF. Therefore, regardless of immune status, ADEM caused by measles encephalitis could occur and be a life-threatening complication.

We administered IVIG, mPSL pulse therapy, and intravenous vitamin A to our patient. Although some reports attest to the efficacy of corticosteroid therapy, (13) IVIG, (4) and ribavirin, (14) specific treatments for measles encephalitis have not been established. Recently, intravenous vitamin A administration has been considered for patients with severe measles, including those with encephalitis. Previous studies associate vitamin A deficiency with fewer measles-specific antibodies and increased infection severity and complications $(15,16)$. Two doses of vitamin A $(200,000$ IU on consecutive days) may reduce mortality rates in children $<2$ years of age (17). We supplemented vitamin A intravenously in our case because his serum vitamin A level was very low. Unfortunately, his level of consciousness did not improve. Therefore, the contribution of vitamin A to the patient's neurological improvement after administration of IVIG and corticosteroid was limited. Further experiences are warranted to evaluate the efficacy of vitamin A in adult patients with severe measles.

The efficacy of TPE in measles patients is controversial. In a previous case report, patients with measles encephalitis (confirmed by IgM antibody positivity in the CSF) recovered consciousness after the application of TPE (18). Currently, TPE is recommended as a therapeutic alternative for several neurological diseases, including ADEM, when corticosteroids are ineffective (19). A previous report explained that TPE removes offending circulating antibodies and cytokines, also affects the $\mathrm{T}$ cell balance in patients with neuroimmunological diseases (20). For now, a robust randomized control trial is lacking. However, the improvement of our patient might have attributed to TPE. New insights on the role of TPE in adult ADEM patients are warranted.

Measles require airborne precaution; the infectious period of measles is several days before and after rash onset. However, in our case, since measles viral RNA had been detected from various specimens other than respiratory secretion such as blood, CSF and urine, the appropriate length of isolation required for his measles encephalitis was not clearly understood. Thus, the patient was isolated in the negative pressure room; medical workers with sufficient measles $\mathrm{IgG}$ titer took care of him and continuously wore an N95 respirator. Every circumstances he touched was disinfected using alcohol or sodium hypochlorite wipes. To determine the isolation period, we performed measles PCR tests of throat swab, urine, and CSF specimens monthly. We terminated isolation when the time the throat swab specimens became negative ( 3 months post-hospitalization). Fortunately, no medical workers contracted measles. Further studies regarding the optimal duration of isolation for patients with measles encephalitis are needed to avoid secondary infections.

In conclusion, we described a case of severe measles encephalitis that occurred in an immunocompetent patient during the 2018 measles outbreak in Japan. The infection of $>170$ people in Okinawa, Aichi, Fukuoka, and Tokyo within 3 months underscores the rapid measles virus infectivity in many parts of Japan, a developed, industrialized country. Measles remains an ongoing infectious disease, though in rare cases, causes its encephalitis and ADEM. Ceaseless efforts are needed to eliminate this public health menace.

The patient provided written, informed consent for the publication of his medical information and clinical images in this report

The authors state that they have no Conflict of Interest (COI).

\section{Acknowledgement}

The authors would like to thank Prof. Susumu Kusunoki (Department of Neurology, Kindai University Medical School) for conducting the analysis of the anti-glycolipid antibodies.

\section{References}

1. Inaida S, Matsuno S, Kobune F. Measles elimination and immunisation: national surveillance trends in Japan, 2008-2015. Epidemiol Infect 145: 2374-2381, 2017.

2. Dabbagh A, Patel MK, Dumolard L, et al. Progress toward regional measles elimination - worldwide, 2000-2016. MMWR Morb Mortal Wkly Rep 66: 1148-1153, 2017.

3. World Health Organization. Measles and rubella surveillance data [Internet]. [cited 2020 Apr 29]. Available from: https://www.who.i $\mathrm{nt} /$ immunization/monitoring_surveillance/burden/vpd/surveillance_t ype/active/measles_monthlydata/en/

4. Fox A, Hung TM, Wertheim H, et al. Acute measles encephalitis in partially vaccinated adults. PLoS One 8: e71671, 2013.

5. National Institute of Infectious Diseases. Infectious agents surveillance report Vol 40. No. 4(No. 470) April 2019 [Internet]. [cited 2020 Apr 29]. Available from: https://www.niid.go.jp/niid/en/iasr-v ol40-e/865-iasr/8754-470te.html (In Japanese).

6. National Institute of Infectious Diseases, Japan. [cited 2020 Apr 29]. Available from: https://www.niid.go.jp/niid/en/y-graphs.html

7. Naim HY. Measles virus. Hum Vaccin Immunother 11: 21-26, 2015.

8. Fisher DL, Defres S, Solomon T. Measles-induced encephalitis. QJM 108: 177-182, 2015.

9. Menge T, Hemmer B, Nessler S, et al. Acute disseminated encephalomyelitis: an update. Arch Neurol 62: 1673-1680, 2005.

10. Moss WJ. Measles. Lancet 390: 2490-2502, 2017.

11. Rafat C, Klouche K, Ricard JD, et al. Severe measles infection: 
the spectrum of disease in 36 critically ill adult patients. Medicine (Baltimore) 92: 257-272, 2013.

12. Kennedy CR, Webster AD. Measles encephalitis. N Engl J Med 311: 330-331, 1984.

13. Bichon A, Aubry C, Benarous L, et al. Case report: ribavirin and vitamin A in a severe case of measles. Medicine (Baltimore) 96: e9154, 2017.

14. Liyanage HJD, Faizal MAM, Kanankearachchi KPP. Management of severe measles related pneumonia, acute respiratory distress syndrome and pleural effusion with intravenous methylprednisolone, immunoglobulin and oral vitamin A. Sri Lanka J Child Health 45: 215-217, 2016.

15. Perry RT, Halsey NA. The clinical significance of measles: a review. J Infect Dis 189 (Suppl): S4-S16, 2004.

16. Frieden TR, Sowell AL, Henning KJ, Huff DL, Gunn RA. Vitamin A levels and severity of measles. New York City. Am J Dis Child 146: 182-186, 1992.

17. Huiming Y, Chaomin W, Meng M, Yang H, Wan C, Mao M. Vita- min A for treating measles in children. Cochrane Database Syst Rev 2005: CD001479, 2005.

18. Hagiwara H, Sakamoto S, Katsumata T, Katayama Y. Acute disseminated encephalomyelitis developed after Mycoplasma pneumoniae infection complicating subclinical measles infection. Intern Med 48: 479-483, 2009.

19. Cortese I, Chaudhry V, So YT, Cantor F, Cornblath DR, Rae-Grant A. Evidence-based guideline update: plasmapheresis in neurologic disorders: report of the Therapeutics and Technology Assessment Subcommittee of the American Academy of Neurology. Neurology 76: 294-300, 2011.

20. Lin CH, Jeng JS, Yip PK. Plasmapheresis in acute disseminated encephalomyelitis. J Clin Apher 19: 154-159, 2004.

The Internal Medicine is an Open Access journal distributed under the Creative Commons Attribution-NonCommercial-NoDerivatives 4.0 International License. To view the details of this license, please visit (https://creativecommons.org/licenses/ by-nc-nd/4.0/).

(C) 2021 The Japanese Society of Internal Medicine Intern Med 60: 1971-1976, 2021 